\title{
CONTINUOUS HOMOMORPHIC IMAGES OF REAL CLANS WITH ZERO $^{1}$
}

\author{
HASKELL COHEN AND I. S. KRULE
}

A clan is a compact connected topological semigroup with identity element. We call a clan a real clan provided its underlying topological space is a closed interval of real numbers with the usual topology. Recently A. D. Wallace conjectured that the continuous homomorphic image of a real clan with zero is again one such. We show that under suitable conditions this conjecture is true, but that in general the image is either a real clan with zero or a triad (i.e., homeomorphic to the letter " $T$ " without serifs); moreover, if the image is a triad, then its zero is an endpoint.

Throughout this paper $S$ denotes a real clan with zero, $u$ its identity, $z$ its zero, and $E$ its set of idempotents, and $h$ is a continuous homomorphism of $S$ onto the clan $T$.

Lemma 1. If $a, b \in S$ and if $a$ separates $b$ and $z$, then there exists $r \in S$ such that $r$ separates $z$ and $u, r b=a$, and $r$ separates $a$ and $u$ or $r=a$.

Proof. The first two conclusions of the lemma are immediate from the continuity of multiplication, and the third conclusion follows from a result of Faucett [2, Lemma 1].

Lemma 2. Let $S=[\delta, u]$, let $C$ be a component of $S \backslash\{z\}$, and let $p$ be the endpoint of $C$. Suppose $a \in \bar{C}$ and $\bar{C} \cap h^{-1} h(a) \neq\{a\}$. If $r \in[z, u]$ with $a=r p$ and if $e=\sup (E \cap\{x \in S \mid x \leqq r\})$, then $h(e p)=h(a)$.

Proof. Let $b \in \bar{C} \cap h^{-1} h(a)$ with $b \neq a$. Then by Lemma 1 there exists $s \in[z, u]$ such that $b=s p$.

CASE 1. $a=z$ or $a$ separates $b$ and $z$. Then $r<s[1$, Lemma 2.5] and from Lemma 1 there exists $t \in[r, u]$ such that $r=t s$, so that $h(a)$ $=h(r p)=h(t s p)=h(t) h(s p)=h(t) h(b)=h(t) h(a)=\cdots=h\left(t^{n}\right) h(a), \quad n$ $=1,2,3, \cdots$. From the structure of the multiplication in $S[3$, Theorem B], from the fact that $[z, u]$ is a subclan of $S[1$, Lemma $2.1]$, then from the continuity of $h$ it follows that $\left\{t^{n}\right\}_{n=1}^{\infty}$ converges to some $f \in E \cap[e, t]$ such that $h(a)=h(f) h(a)$. We assert $e=f$. For if $e<f$ and $r<f$. In this case if $f \leqq s$ then $r<f \leqq t s$ [3, Theorem B], a contradiction, while if $s<f$ then $r<s=t s$ [3, Theorem B], again a

Presented to the Society, April 19, 1958; received by the editors April 14, 1958.

1 The work of one of the authors was partially supported by the United States Air Force under Contract No. AF 18(603)-89 monitored by the AF Office of Scientific Research of the Air Research and Development Command. 
contradiction. Therefore $e=f$, and $h(a)=h(f) h(a)=h(e) h(r p)=h(e r p)$ $=h(e p)[3$, Theorem B].

CASE 2. $b=z$ or $b$ separates $a$ and $z$. Then $s<r$ [1, Lemma 2.5]. If $e \leqq s$ then $e=\sup (E \cap\{x \in S \mid x \leqq s\})$, and Case 1 gives $h(e p)=h(b)$ $=h(a)$. If $s<e$ then again using [3, Theorem B] we have $h(e p)$ $=h(e r p)=h(e a)=h(e) h(a)=h(e) h(b)=h(e) h(s p)=h(e s p)=h(s p)$ $=h(b)=h(a)$.

LEMma 3. If $C$ is a component of $S \backslash\{z\}$ and if $\bar{h}$ is the restriction of $h$ to $\bar{C}$, then $\bar{h}$ is monotone. ${ }^{2}$

Proof. Say $S=[\delta, u]$, and let $p$ be the endpoint of $C$. Suppose that $a, b, c \in \bar{C}$ with $h(a)=h(b)$ and $c$ between $a$ and $b$. It then suffices to show that $h(c)=h(a)$. Without loss of generality we assume $a=z$ or $a$ separates $b$ and $z$. By Lemma 1 and [1, Lemma 2.5] there exist $r, s, t \in[z, u]$ such that $r<t<s, r p<a, s p=b$, and $t p=c$. Let $e=\sup (E \cap\{x \in S \mid x \leqq r\})$; from Lemma 2 we have $h(a)=h(e p)$ $=h(b)$. By Lemma 1 there exists $y \in[t, u]$ such that $y s=t$. Thus we get $h(c)=h(t p)=h(y s p)=h(y b)=h(y) h(b)=h(y) h(e p)=h(y e p)=h(e p)$ $=h(a)$.

As an immediate consequence (see [4, p. 165]) of Lemma 3 or from Theorem 2(i) below we have

THEOREM 1. If $z$ is an endpoint of $S$, then $T$ is a real clan with zero, and its zero is an endpoint.

Lemma 4. Let $S=[\delta, u]$. Suppose $t_{0} \in T$ and $h^{-1}\left(t_{0}\right)$ meets both $[\delta, z)$ and $(z, u]$. If $a=\inf h^{-1}\left(t_{0}\right)$ and $b=\sup h^{-1}\left(t_{0}\right)$, then $h([a, z])$ $=h([z, b])$.

Proof. If $x \in[a, z]$, then by Lemma 1 there exists $r \in[z, u]$ such that $x=r a$. Now $r b \in[z, b]$, and $h(r b)=h(r) h(b)=h(r) h(a)=h(r a)$ $=h(x)$. Hence $h([a, z]) \subset h([z, b])$. A similar demonstration shows $h([z, b]) \subset h([a, z])$.

Theorem 2. Let $S=[\delta, u]$, and let $D$ be the set of those $x \in S$ for which $h^{-1} h(x)$ meets both $[\delta, z)$ and $(z, u]$.

(i) If $D$ is void, then $T$ is a real clan with zero, and its endpoints are $h(u)$ and $h(\delta)$.

(ii) If inf $D=\delta$, then $T$ is a real clan with zero, and its endpoints are $h(u)$ and $h(z)$.

(iii) If $\delta<\inf D<z$ and $h(D)=h(z)$, then $T$ is a real clan with zero and its endpoints are $h(u)$ and $h(\delta)$.

' A function $f$ on a space $X$ to a set $Y$ is said to be monotone provided $f^{-1}(y)$ is connected for each $y \in Y$. 
(iv) If $\delta<\inf D<z$ and $h(D) \neq h(z)$, then $T$ is a triad and its endpoints are $h(u), h(\delta)$, and $h(z)$.

Proof. Let $D_{0}=D \cup\{z\}$. Since $h$ is continuous, $D_{0}$ is closed; and if $D$ is not void, then it follows from Lemma 4 that $D_{0}=\bar{D}$.

(i) Clearly, $D$ is void implies $h$ is monotone, so that $[4$, p. 165] $T$ is a real clan with zero, and its endpoints are $h(u)$ and $h(\delta)$.

(ii) If inf $D=\delta$, then it follows from Lemma 4 that $h(S)=h([z, u])$. But $[z, u]$ is a subclan of $S[1$, Lemma 2.1$]$, and therefore Theorem 1 applies to $[z, u]$, giving the desired conclusion.

(iii) and (iv). Let $a=\inf D$ and $b=\sup D$. Now $h(a)=h(b)$ so that from Lemmas 3 and 4 we see $h(S)=h([\delta, a]) \cup h\left(D_{0}\right) \cup h([b, u])$, $h([\delta, a]) \cap h\left(D_{0}\right)=h(a), h\left(D_{0}\right) \cap h([b, u])=h(b), h([\delta, a]) \cap h([b, u])$ $=h(a)$, and $h$ restricted to each of $[\delta, a], D_{0}$, and $[b, u]$ is monotone. Further from Lemma 4 we have $h\left(D_{0}\right)=h([a, z])$, and thus $T$ is the union of the $\operatorname{arcs} h\left(D_{0}\right)$ (possibly $\left.h(z)\right), h([\delta, a])$, and $h([b, u])$ which pairwise have intersection $h(a)$. Consequently (iii) and (iv) follow.

We observe that the cutpoint properties of $T$ are independent of the metrisability of $S$, so that a theorem analogous to Theorem 2 obtains if $S$ is a clan with zero irreducibly connected between some two of its points.

The following example illustrates the case considered in Theorem 2 (iii).

EXAMPLE. Let $S=[-1,1]$ with the usual multiplication and topology, and let $T$ be $[-1,-1 / 2) \cup[0,1]$ with the canonical topology induced by the following function $h$ from $S$ onto $T$ :

$$
h(x)=\left\{\begin{aligned}
x & \text { if } 0 \leqq x \text { or } x<-1 / 2 \\
-x & \text { if }-1 / 2 \leqq x<0
\end{aligned}\right.
$$

The multiplication "O" in $T$ is defined as follows, where $a b$ means the usual product:

$$
a \circ b=\left\{\begin{aligned}
a b & \text { if } 0 \leqq a b \text { or } a b<-1 / 2, \\
-a b & \text { if }-1 / 2 \leqq a b<0
\end{aligned}\right.
$$

Then $h$ is a continuous homomorphism of the real clan with zero $S$ onto the clan with zero $T$, which is a triad.

\section{REFERENCES}

1. Haskell Cohen and L. I. Wade, Clans with zero on an interval, Trans. Amer. Math. Soc. vol. 88 (1958) pp. 523-535.

2. W. M. Faucett, Compact semigroups irreducibly connected between two idempotents, Proc. Amer. Math. Soc. vol. 6 (1955) pp. 741-747. 
3. P. S. Mostert and A. L. Shields, On the structure of semigroups on a compact manifold with boundary, Ann. of Math. vol. 65 (1957) pp. 117-143.

4. G. T. Whyburn, Analytic topology, Amer. Math. Soc. Colloquium Publications, vol. 28, 1942, New York.

Louisiana State University

\section{ON GREEN'S THEOREM}

PAUL J. COHEN

Green's theorem in two dimensons says that if $C$ is a simple closed curve bounding the region $Q$, if $A(x, y)$ and $B(c, y)$ are continuous functions having derivatives, then under suitable further conditions we have,

$$
\int_{C} A d x+B d y=\iint_{Q}\left(\frac{\partial B}{\partial x}-\frac{\partial A}{\partial y}\right) d x d y
$$

where the line integral is taken in a positive sense around the curve $C$. In [1] Bochner investigated under which conditions (1) holds. There it was shown that if $A$ and $B$ have certain regularity properties and if the integrand on the right of (1) behaves well, then (1) does hold. Here we shall prove (1) under what may be regarded as the weakest possible hypotheses. This question was also treated by Shapiro in [3], and though he assumes certain regularity of $A$ and $B$, namely, the existence of the differential, he allows certain exceptional sets which we cannot allow. The proof of our theorem is modeled after the proof of the Looman-Mensov theorem as contained, for example, in [2]. We will not deal with the topological difficulties involved so that our theorem will only treat the case in which $Q$ is a rectangle, whose sides are parallel to the coordinate axes.

Theorem. Let $A(x, y)$ and $B(x, y)$ be two functions defined on the rectangle $Q$, and continuous on the closure of $Q$. Assume further that the partial derivatives

$$
\frac{\partial A}{\partial x}, \frac{\partial A}{\partial y}, \frac{\partial B}{\partial x}, \frac{\partial B}{\partial y}
$$

exist everywhere in the interior of $Q$, except perhaps at a countable num-

Presented to the Society, April 26, 1958; received by the editors March 27, 1958. 